\title{
DOD-DOA Estimation Based on Cyclic Statistics of Bistatic MIMO Radar
}

\author{
Xiaoying $\mathrm{Li}^{1, \mathrm{a}^{*}}$, Hanying $\mathrm{Hu}^{1, \mathrm{~b}}$, Yilun $\mathrm{Chen}_{\mathrm{e}}^{1, \mathrm{c}}$, Yinghua Tian ${ }^{1, \mathrm{~d}}$ and Song Chen ${ }^{1}$ \\ ${ }^{1}$ Zhengzhou Information Science and Technology Institute, Zhengzhou 450000, China; \\ abailuoboyingying@126.com, ${ }^{b}$ huhanying@vip.sina.com, ${ }^{c} 1024169662 @ q q . c o m,{ }^{d}$ tian.ying.hua@f \\ oxmail.com, ${ }^{\mathrm{e}}$ wirelessmancs@163.com
}

Keywords: MIMO radar, broadband signal, DOD-DOA estimation, cyclostationary.

\begin{abstract}
Focused on the DOD-DOA estimation of bistatic MIMO radar, we present a method using cyclostationary broadband signal to process MUSIC algorithm. More information is carried in broadband signal and its cyclostationary property restrains Gaussian noise as well as interference from different frequencies effectively. The result of simulation shows that the method has high angel resolution and signal selectivity.
\end{abstract}

\section{Introduction}

In recent years, MIMO (Multiple-input Multiple-output) radar has gotten considerable attention[1, 2, 3]. It extends signal channel through virtual array formed by multiple transmit array and receiving array. MIMO radar could achieve space diversity and waveform diversity.

Many artificial signals, as well as natural signals, have cyclostationary characters in real environment like television signal, communication signal and radar signal[4]. Cyclostationary theory has become a hot research topic. In recent years, people start to applying the space-time characteristic of signal to radar angle estimation and presented algorithms like Cyclic MUSIC、Cyclic ESPRIT and CLS, which can be called generally by Cyclic-SSF algorithm. And it is superior to the former spatial spectrum method in aspects like signal selectivity, overload capacity and signal detection, etc. In reference [5], Cyclic-MUSIC algorithm was used to improve DOA estimation accuracy of MIMO radar; Reference [6] made use of cyclostationary characters of signal and ESPRIT algorithm to estimate DOD-DOA of bistatic MIMO radar. However, the premise of these algorithms is that the signal is a narrow band signal; for broadband signal, it will produce a much larger estimation error, though broadband signal can carry more information compared to narrow band signal and therefore is used widely in signal detection, parameter estimation, target tracking, etc[7]. For broadband signal, each frequency corresponds to different steering vectors and further on, different signal subspace, while cyclic autocorrelation function retain phase information. $\mathrm{Xu}$ et al. used this character and proposed SC-SSF for broadband signal DOA estimation[8]. Reference [9] constructed cyclostationary correlation matrix and conjugate cyclostationary correlation matrix at the same time, then combined regular MUSIC to estimate the DOA of MIMO radar.

In this contribution, we will focus on angel estimation of bistatic MIMO radar by broadband cyclostationary signal and present an algorithm which restrains Gaussian noise as well as interference from different frequencies effectively. We will start by giving a brief introduction to cyclostationary signal, then analyze bistatic MIMO radar model and calculate the circular correlation matrix of received signal, finally estimate angel by MUSIC algorithm and conduct simulation of the algorithm.

\section{Basic Concepts of Cyclostationarity Signals}

A process of which statistical properties change periodically or cyclostationarily is called cyclostationary process. For a signal $s(t)$, if its first-order and second-order statistical properties show a certain pattern during a period of time, then it can be called generalized cyclostationary signal[10]. That is: 


$$
\left\{\begin{array}{c}
m_{s}(t)=m_{s}(t+T) \\
R_{s}(t, \tau)=R_{s}(t+T, \tau)
\end{array} .\right.
$$

Where $T=K / \alpha$ and $K$ is integer, $\alpha$ denotes cycle frequency, $m_{s}(t)=E[s(t)]$ is the average value of the signal, $R_{s}(t, \tau)=E\left[s(t+\tau / 2) s^{*}(t-\tau / 2)\right]$ is the autocorrelative function of the signal.

Generally, $s(t)$ is a periodic ergodic process. So we can obtain

$$
\begin{aligned}
R_{s}^{\alpha}(\tau) & =\lim _{T \rightarrow \infty} \int_{-T / 2}^{T / 2} s(t+\tau / 2) s^{*}(t-\tau / 2) e^{-j 2 \pi \alpha t} d t \\
& =\left\langle s(t+\tau / 2) s^{*}(t-\tau / 2) e^{-j 2 \pi \alpha t}\right\rangle
\end{aligned} .
$$

Where \langle\rangle is the notation of getting the average of all time, $R_{s}^{\alpha}(\tau)$ is the autocorrelative function of $s(t)$.

\section{Signal Model and Angle Estimation}

Consider a centralized bistatic MIMO radar system shown in Fig. 1, with an M-element transmit array and an N-element receiving array. Both of them are uniform linear arrays (ULAs) and all the elements are omnidirectional. The distance between the antenna elements in transmit array and receiving array are noted by $d_{t}$ and $d_{r}$. Each of the transmit elements transmits orthogonal waveforms with the same carrier frequency and the same bandwidth at the same time, which denotes $S(t)=\left[s_{1}(t), s_{2}(t), \cdots, s_{M}(t)\right]^{T}$. Assume that there are a number of $\mathrm{D}$ uncorrelated distal point targets at space. And the position of the $k$ th $(k=1,2, \cdots, D)$ target is denoted as $\left(\theta_{k}, \varphi_{k}\right)$, where the $\theta_{k}$ denotes the direction of departure (DOD) and the $\varphi_{k}$ denotes the direction of arrival (DOA) of the signal with respect to the $k$ th target.

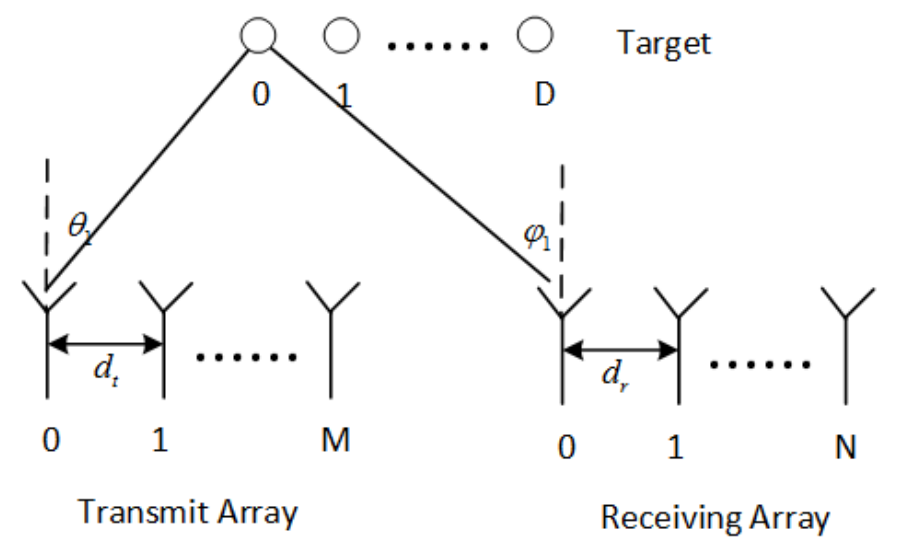

Fig. 1 Bistatic MIMO radar system

Assuming there is a single target at position $\left(\theta_{k}, \varphi_{k}\right)$, the signal vector of the receiving array can be written as:

$$
y_{p}(t)=\xi_{k} \sum_{m=1}^{M} s_{m}\left[t+(p-1) \tau_{b k}+(m-1) \tau_{a k}\right]+w_{p k}(t) .
$$

Where $\tau_{a k}=d_{t} / \lambda \sin \left(\theta_{k}\right)$ and $\tau_{b k}=d_{r} / \lambda \sin \left(\varphi_{k}\right)$ are time delay of signal on the adjacent transmit elements and receiving elements, respectively, $\lambda$ is the wavelength, $w_{p k}(t)$ is the additive white Gaussian noise ( AWGN) and interference vector on the $p$ th $(p=1,2, \cdots, N)$ receiving element. 
Due to the better performance that cyclostationary signal possesses at the parameter estimation, it not only can restrain Gauss noise, but also resist interference. Thus it is used in the autocorrelation matrix's construction of the signal. According to the definition of the cyclostationary function, the cyclostationary function between the $p$ th receiving element and the $q$ th receiving element could be shown as:

$$
\begin{aligned}
R_{y_{p} y_{q}}^{\alpha}(\tau)= & \left\langle y_{p}(t+\tau / 2) y_{q}^{*}(t-\tau / 2) e^{-j 2 \pi \alpha t}\right\rangle \\
= & \left\langle\xi_{k} \xi_{k}^{*} \sum_{m=1}^{M} s_{m}\left[t+(m-1) \tau_{a k}+(p+q-2) \tau_{b k} / 2+\left((p-q) \tau_{b k}+\tau\right) / 2\right] .\right. \\
& \left.s_{m}^{*}\left[t+(m-1) \tau_{a k}+(p+q-2) \tau_{b k} / 2-\left((p-q) \tau_{b k}+\tau\right) / 2\right] e^{-j 2 \pi \alpha t}\right\rangle
\end{aligned}
$$

For notational convenience: $s_{m}^{\prime}(t)=s_{m}\left[t+(m-1) \tau_{a k}+(p+q-2) \tau_{b k} / 2\right]$, the Eq.4 could be written as:

$$
R_{y_{p} y_{q}}^{\alpha}(\tau)=\xi_{k} \xi_{k}^{*} \sum_{m=1}^{M} R_{s_{m}^{\prime} s_{m}}^{\alpha}\left[(p-q) \tau_{b k}+\tau\right] .
$$

According to the definition of the cyclostationary function, let $s^{\prime}(t)=s\left(t+\tau_{d}\right)$, so we can get:

$$
\begin{aligned}
R_{s^{\prime} s^{\prime}}^{\alpha}(\tau) & =\left\langle s^{\prime}(t+\tau / 2) s^{* *}(t-\tau / 2) e^{-j 2 \pi \alpha t}\right\rangle \\
& =\left\langle s\left(t^{\prime}+\tau / 2\right) s^{*}\left(t^{\prime}-\tau / 2\right) e^{-j 2 \pi \alpha t^{\prime}} e^{j 2 \pi \alpha \tau_{d}}\right\rangle . \\
& =R_{s s}^{\alpha}(\tau) e^{j 2 \pi \alpha \tau_{d}}
\end{aligned}
$$

So, the formula Eq.5 can be rewritten as:

$$
R_{y_{p} y_{q}}^{\alpha}(\tau)=\xi_{k} \xi_{k}^{*} \sum_{m=1}^{M} R_{s_{m} s_{m}}^{\alpha}\left[(p-q) \tau_{b k}+\tau\right] e^{j 2 \pi \alpha\left((m-1) \tau_{a k}+(p+q-2) \tau_{b k} / 2\right)} .
$$

When there are $D$ targets with complex random variables $\xi_{k}(k=1,2, \ldots, D)$, as $\xi_{k} \sim C N(0,1)$, the signals of the $p$ th receiving element are

$$
\begin{aligned}
y_{p}(t) & =\sum_{k=1}^{D}\left\{\xi_{k} x_{k}\left[t+(p-1) \tau_{b k}\right]+w_{p k}(t)\right\} \\
& =\sum_{k=1}^{D} \xi_{k}\left\{\sum_{m=1}^{M}\left\{s_{m}\left[t+(p-1) \tau_{b k}+(m-1) \tau_{a k}\right]+n_{k m}(t)\right\}+w_{p k}(t)\right\}
\end{aligned}
$$

Then, we obtain the cyclostationary function as:

$$
\begin{gathered}
R_{y_{p} y_{q}}^{\alpha}(\tau)=\sum_{k_{1}=1}^{D} \sum_{k_{2}=1}^{D} \sum_{m=1}^{M} \xi_{k_{1}} \xi_{k_{2}}^{*} R_{s_{m} s_{m}}^{\alpha}\left[(p-1) \tau_{b k_{1}}-(q-1) \tau_{b k_{2}}+(m-1)\left(\tau_{a k_{1}}-\tau_{a k_{2}}\right)+\tau\right] . \\
e^{j \pi \alpha\left((p-1) \tau_{b k_{1}}+(q-1) \tau_{b k_{2}}+(m-1)\left(\tau_{a k_{1}}+\tau_{a k_{2}}\right)\right)} .
\end{gathered}
$$

The cyclostationary function we obtained from Eq.10 is independent of the signal bandwidth. Therefore, it is suitable for both narrowband and broadband signals. The information of DOD and DOA of the signals is contained in the cyclostationary function, thus we can construct a $N \times N$ matrix $R_{Y}^{\alpha}(\tau)$ as follows

$$
R_{Y}^{\alpha}(\tau)=\left[\begin{array}{cccc}
R_{y_{1} y_{1}}^{\alpha}(\tau) & R_{y_{1} y_{2}}^{\alpha}(\tau) & \cdots & R_{y_{1} y_{N}}^{\alpha}(\tau) \\
R_{y_{2} y_{1}}^{\alpha}(\tau) & R_{y_{2} y_{2}}^{\alpha}(\tau) & \cdots & R_{y_{2} y_{N}}^{\alpha}(\tau) \\
\vdots & \vdots & \ddots & \vdots \\
R_{y_{N} y_{1}}^{\alpha}(\tau) & R_{y_{N} y_{2}}^{\alpha}(\tau) & \cdots & R_{y_{N} y_{N}}^{\alpha}(\tau)
\end{array}\right]
$$

Correcting the matrix $R_{Y}^{\alpha}(\tau)$ [11], we have 


$$
R(\alpha)=\frac{1}{2 L-1} \sum_{\tau=-L+1}^{L-1} R_{Y}^{\alpha}(\tau)
$$

Using the singular value decomposition (SVD) on the cyclic autocorrelation matrix $R(\alpha)$ we can obtain the signal subspace $U_{S}^{\alpha}$ and noise subspace $U_{N}^{\alpha}$.According to the MUSIC algorithm we can get the DOD and DOA of the signals.

Based on the analysis above, the algorithm can be concluded in the following steps:

Step 1: Calculate the cyclostationary function $R_{y_{p} y_{q}}^{\alpha}(\tau)(p, q=1,2, \cdots, N)$ with respect to $y_{p}(t)(p, q=1,2, \cdots, N)$ obtained from receiving array.

Step 2: $\quad$ Construct the matrix $R_{Y}^{\alpha}(\tau)$ with a dimension of $N \times N$ by the $R_{y_{p} y_{q}}^{\alpha}(\tau)$.

Step 3: $\quad$ Correct $R_{Y}^{\alpha}(\tau)$ and get the matrix $R(\alpha)=\frac{1}{2 L-1} \sum_{\tau=-L+1}^{L-1} R_{Y}^{\alpha}(\tau)$.

Step 4: $\quad$ Compute the singular value decomposition (SVD) of $R(\alpha)$ and obtain the signal subspace $U_{S}^{\alpha}$ and noise subspace $U_{N}^{\alpha}$.

Step 5: $\quad$ Obtain the DOD and DOA by MUSIC algorithm.

\section{Experiment Simulation}

To verify the algorithm, we conduct some experiments in the mixed environment of additive Gauss colored noise with a signal-to-noise ratio(SNR)of $5 \mathrm{~dB}$ and an $5 \mathrm{~dB}$ interference signal, of which cycle frequency differs from that of transmit signals. The radar system is a bistatic MIMO radar with $M=5$ transmit array elements and $N=4$ receiving array elements. Each element space is half-wavelength. The transmit array transmits the broadband BPSKs with the same cycle frequency at the same time. The frequency of the MIMO radar is $20 \mathrm{MHz}$, and the cycle frequency of transmit signal and interference signal are $\alpha=0.4$ and $\alpha=0.6$, respectively. The number of snapshots is 512 , and we take the average of $P=100$ Monte Carlo trials as the experimental result. Assuming that there are three uncorrelated targets located at $\left(\theta_{1}, \varphi_{1}\right)=\left(12^{\circ},-34^{\circ}\right) 、\left(\theta_{2}, \varphi_{2}\right)=\left(-73^{\circ}, 49^{\circ}\right)$ and $\left(\theta_{3}, \varphi_{3}\right)=\left(45^{\circ}, 60^{\circ}\right)$, and the interference signal affects the receive array with an $55^{\circ}$ incidence angle. Each target has the reflection coefficient as $\zeta_{1}=0.65 、 \zeta_{2}=0.8$ and $\zeta_{3}=0.73$.

Trial 1: Verification on the validation of the algorithm. Fig. 2 shows the spectrum of the estimated DOD and DOA of the targets. Fig. 3 shows the accurate estimation of the DOD and DOA. The estimated DOD and DOA is highly in accordance with the true value, thus we can prove the algorithm is of good performance.

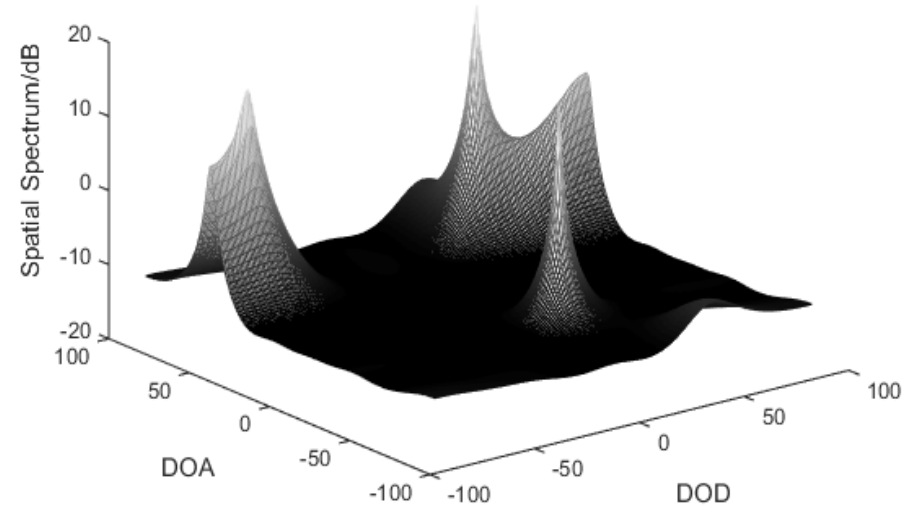

Fig. 2 The spectrum of the estimated DOD and DOA of the targets 


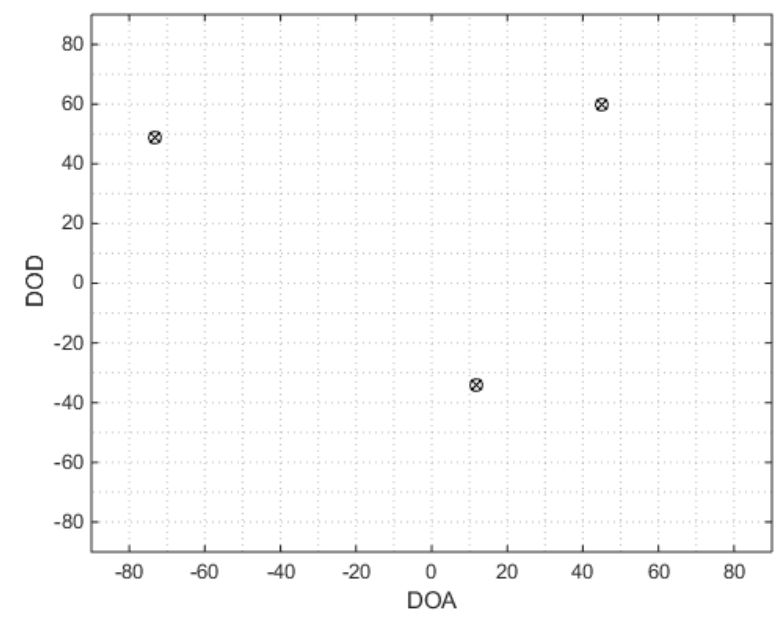

Fig. 3 The accurate estimation of the DOD and DOA

Trial 2: Verification on the performance of the algorithm. Compared with the traditional MUSIC algorithm, we use the root mean squared errors (RMSE) to reflect the performance of the algorithm. Due to the signal-to-noise ratio (SNR) of signal influences the performance of the algorithm directly, we will observe the changing of RMSE under different SNR. According to the definition of the RMSE, we obtain its expression as follows referring to the reference [6]

$$
R M S E=\sqrt{\frac{1}{D P} \sum_{k=1}^{D} \sum_{i=1}^{P}\left\{\left(\hat{\theta}_{k i}-\theta_{k}\right)^{2}+\left(\hat{\varphi}_{k i}-\varphi_{k}\right)^{2}\right\}} .
$$

Fig. 4 shows the RMSE of the DOD and DOA estimation changing versus SNR from 100 Monte Carlo trials. It indicates that the cyclic MUSIC algorithm proposed in this paper is better than the traditional MUSIC algorithm when the environment exist colored Gauss noise and interference signals.

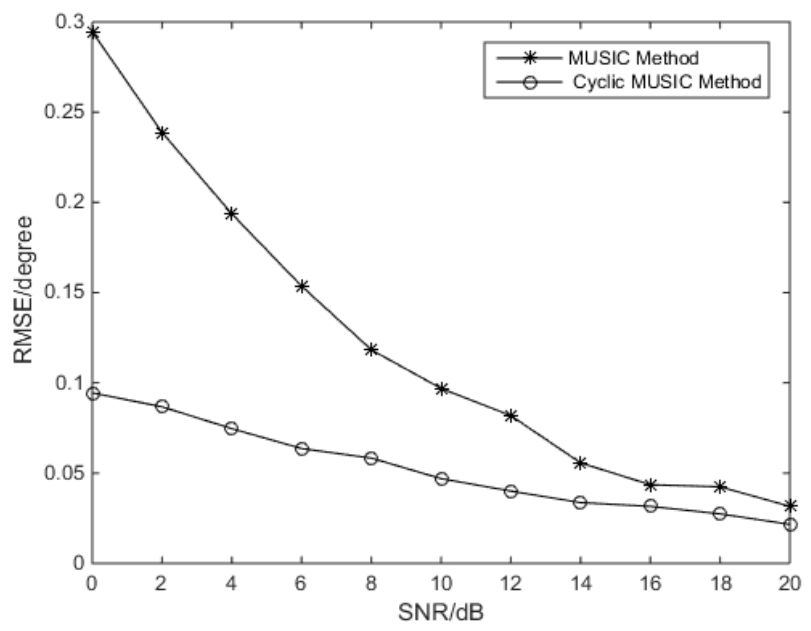

Fig. 4 RMSE of the DOD and DOA estimation versus SNR

\section{Summary}

The algorithm we presented canrestrainGaussian noise as well as interference from different frequencies effectively, which achieves DOD/DOA estimation and increases resolution ratio. The disadvantage is that we have to obtain cycle frequency of the signal precisely for that it directly affects estimation precision, so this algorithmcan only be used for active radar. 


\section{References}

[1] J. Li, P. Hasegawa, and P. Stoica, “Array signal processing for MIMO radar,” Wiley, 2009.

[2] Haimovich A M, Blum R S, Cimini L J. MIMO Radar with Widely Separated Antennas[J]. IEEE Signal Processing Magazine, 2008, 25(1):116-129.

[3] Fishler E, Haimovich A, Blum R S, et al. Spatial diversity in radars-models and detection performance [J]. IEEE Transactions on Signal Processing, 2006, 54(3):823-838.

[4] Chen H, Wang Y, Wang B H. The pre-processing method based on signal conjugate cyclostationary[C]// Antennas and Propagation Society International Symposium, 2003. IEEE. IEEE, 2003:280 - 283.

[5] Wang Y, Gao Y, Jiang H, et al. DOA Estimation of MIMO Radar System Based on Cyclic Statistics[C]// Wireless Communications, Networking and Mobile Computing (WiCOM), 2012 8th International Conference on. IEEE, 2012:1 - 4.

[6] Yu C, Zhang X, Bai Y, et al. DOD-DOA estimation by exploiting signal cyclostationarity for bistatic MIMO radar[C]// Signal Processing, Communication and Computing (ICSPCC), 2013 IEEE International Conference on. IEEE, 2013:1-4.

[7] Liu F G. The Research on Direction of Arrival Estimation of Wide-Band Signals [D]. Haibin Engineering University, 2013.

[8] Wang Y, Yin Q, Jin L, et al. Estimation DOA of Correlated Signal via Modified SC SSF Method [J]. Acta Electronica Sinica, 1999.

[9] Liu F G, Diao M. DOA Estimation of Wideband Signals Based on Cyclostationarity [J]. Journal of Applied Sciences, 2012.

[10]Zhang C, Chen D, Lu H, et al. Cyclic Cross-correlation based Two-dimensional Direction of Arrival Estimation Algorithm[J]. IEEE, 2008:1148-1152.

[11]Wang Y, Zu-Kun L U, Gao Y, et al. Estimation of DOA Based on Cyclic-Music Algorithm in MIMO Radar System [J]. Journal of Jilin University, 2012, 30(4). 